\title{
Experimental Study of the Aerosolization from a Carbon Nanotube Bulk by a Vortex Shaker
}

\author{
Olivier Louis Claude Le Bihan, ${ }^{1}$ Aurélien Ustache, ${ }^{1}$ Daniel Bernard, ${ }^{2}$ \\ Olivier Aguerre-Chariol, ${ }^{1}$ and Martin Morgeneyer $^{3}$ \\ ${ }^{1}$ Institut National de l'Environnement Industriel et des Risques (INERIS), 60550 Verneuil-en-Halatte, France \\ ${ }^{2}$ ARKEMA, 92705 Colombes, France \\ ${ }^{3}$ Université de Technologie de Compiègne (UTC), 60200 Compiègne, France \\ Correspondence should be addressed to Olivier Louis Claude Le Bihan; olivier.le-bihan@ineris.fr
}

Received 9 December 2013; Revised 11 March 2014; Accepted 11 March 2014; Published 8 May 2014

Academic Editor: Prashant Kumar

Copyright (C) 2014 Olivier Louis Claude Le Bihan et al. This is an open access article distributed under the Creative Commons Attribution License, which permits unrestricted use, distribution, and reproduction in any medium, provided the original work is properly cited.

\begin{abstract}
The growing use of nanomaterials requires the development of tools enabling study of the risks to consumer, worker, and environment. This study relates to the risk of suspension of inhalable particles upon production and/or use of powders constituted of nanoobjects, and more specifically to the potential of the vortex shaker as apparatus for determining the dustiness of a powder and as atmosphere generating tool for experimental toxicology. The powder chosen for this study was Graphistrength C100 (ARKEMA), a multiwalled carbon nanotube. Its agitation in a vortex shaker at $1500 \mathrm{rpm}$ leads to an aerosol divided into four families, from isolated fibres to micronic pellets. The study highlights that the speed of agitation and the geometry of the device are influential parameters, to be systematically taken into account. It concludes that while the technique seems mature to conduct C100 dustiness tests, developments are still necessary to use it routinely for toxicology studies.
\end{abstract}

\section{Introduction}

1.1. The Need for Aerosol Production and Characterization. Nanomaterials offer a potential for innovation that explains their growing use. However, their use presents risks to the health and to the environment. Consequently these risks must be taken into account. Among these risks is the possibility of suspension of particles upon handling nanopowders, namely, aerosolization. In this paper, nanopowder refers to a powder constituted of "nanoobjects" within the meaning of standard ISO 80004 [1]. The study presented here covers two particular aspects of this problem, being the dustiness of the powders firstly and the development of atmospheres enabling the realisation of toxicological studies by inhalation secondly.

Dustiness refers to the propensity of materials to produce airborne dust during handling [2]. This dust is composed of different categories of particles; we can notably distinguish individual primary particles from the agglomerates that they can constitute. In the case in question in this study (carbon nanotubes (CNTs)), and as we will see in the "results" part, primary particles (individual CNTs), agglomerates combining several CNTs, and even pellets can be observed. The measurement of this dustiness makes it possible to conduct actions firstly with regard to risk management (occupational and consumer exposure and release into the environment) and secondly in terms of operating cost (loss of matter). This is why various authors have already sought to determine the dustiness of the different nanopowders and to classify them according to this criterion $[3,4]$.

The toxicological study of the impact of nanoparticle aerosols, whether using in vitro or in vivo approaches, has stimulated the adaptation of existing tools and the development of new devices to create atmospheres integrating this new pollutant (e.g., [5-7]).

For both dustiness tests and development of tests for toxicology, a major issue is the representativeness of the method chosen. Indeed, the aerosolization of a powder is closely linked to two factors: the properties of this powder 
(product properties) and the characteristics of the process (deliberate or not) causing this aerosolization. There are many of these processes [8]: mixing, coating, pneumatic drying, conveying, loading-uploading, bagging, and so forth. Here representativeness refers to the capacity of an aerosolization device to represent as faithfully as possible, in the laboratory, a real situation encountered in the field.

However, while this approach is desirable, there is still a great deal to do in respect of identifying the relevant and measurable parameters, which can lead to a factual evaluation of this concept of representativeness.

To date, the methods of aerosolization are based on known principles such as fall of powder, rotation of a cylinder, and agitation of a tube. Consequently we must seek to establish a link between a precise principle and a situation encountered in the field. A recent normative document thus sought to establish a relationship between given laboratory methods and given real situations [8].

Furthermore, the parametric study of the methods makes it possible to draw teachings. Morgeneyer et al. [9] thus studied - for a given powder - the influence on the emissivity of the mass of the sample and the speed of agitation of a vortex shaker, which led them to make recommendations on the conditions of implementation of this device.

In addition to the issue of representativeness with regard to the real situation, a number of needs have been identified linked to the exploitation of the experimental systems. For the two objectives of use (dustiness and toxicology), it is important to have a repeatable method, which implements measures recognised by the current legislation (mass concentration and different size fractions, e.g., inhalable fraction) and which are easy-to-use and of low cost. The low-cost measure requires that the test remains limited to the use of a reasonable quantity of product. For toxicological tests we can add (1) the absence of transformation of the product (e.g., change of granulometry owing to grinding of the powder), (2) the absence of contamination with a third product that could interfere with the tests and which could be generated by the method (e.g., metal aerosol derived from an apparatus), and (3) the need to be able to choose a given concentration, which (4) is stable during the selected period of exposure.

1.2. State of the Art of Aerosol Production Systems. The interest in particle aerosolization devices is not new since we can reference works from the early 20th century, for example, in 1939 "single-drop" tests by Andreasen et al. [10]. These methods were then perfected and mechanised, for example, by Wright [11]. Their implementation and their adaptation for nanopowders appeared during the 2000s.

In the rest of this section, the authors focus their work on the various needs cited in the previous paragraph.

The standards-old [12] or recent [2] - relating to the characterization of the dustiness of powder by "singledrop" or "rotary drum" use quantities of product that are too high for them to be applied to nanopowders. Jensen et al. [13] consequently chose to miniaturise the tests of the European standard [2] for use of only $6 \mathrm{~g}$ nanopowders of bentonite and organoclay. O'Shaughnessy et al. [4] perfected this approach, managing to use only $15 \mathrm{mg}$ nanopowders of SWCNT, $\mathrm{Al}_{2} \mathrm{O}_{3}$, and others. However, the aerosolization via these miniaturised tests only lasted one to two minutes.

Other more complex tests were implemented, such as the fluidized bed to study the aggregation behaviour of silica powder and potato starch [14], or a fluidized bed of MWCNT [15], completed firstly with stainless steel beads and secondly upstream by a rotary brush supplying the test with aerosol. However, this latter study well illustrates that it is difficult to guarantee, for many methods, that the product is not transformed by the processes implemented. This issue arises particularly for setups using grinding upstream of the aerosolization process, such as a knife-mill for SWCNT [5] or a ball-mill combined with a rotary grater for MWCNT [7].

The last possibility listed here consists of the aerosolization of a sample of product by an agitator. Fujitani et al. [6] chose, for example, to fit a cyclone containing $0.24 \mathrm{~g}$ MWCNT and $5 \mathrm{~mm}$ stainless steel beads on a sieve agitator. While this assembly made it possible to obtain an aerosol for 5 hours, it however proves that the presence of metal beads is essential. A "vortex" agitator was used to examine SWCNT in the presence of micronic bronze spheres, over much shorter test durations, by Maynard et al. [16]. Ogura et al. [3] manage to aerosolise $1 \mathrm{~cm}^{3}$ SWCNT, MWCNT, and other nanopowders with a vortex shaker for 31 minutes, without the presence of foreign bodies (e.g., spheres) in the device. They carried out all of their tests at the rotation speed of $2500 \mathrm{rpm}$. Furthermore, they correlate the test results with field studies at the workplace.

1.3. Experimental Choices and Objectives of This Study. The state of the art and a preliminary parametric study conducted by these authors with a micronic powder of bimodal granulometry [9] suggest that the vortex shaker is one of the best positioned techniques given the requirements expressed in Section 1.1.

Indeed, we can firstly raise the possibility of using a limited quantity of product, with a reasonable cost in respect of investment and use. Furthermore, the representativeness in relation to the processes has been examined within standard ISO 12025 [8]: it is considered (annex A) that the vortex shaker method could (quite) well represent the properties of a relatively wide spectrum of processes.

The tests conducted in this study were conducted without foreign body, which presents a risk of modification and contamination of the product. A parametric study of the vortex shaker, conducted for a micronic powder [9], demonstrated the risk of transformation of the granulometry of the powder, with this risk able to be reduced in a moderated speed range of less than $1800 \mathrm{rpm}$. These parameters potentially depend on the product used. Consequently a study of sensitivity of the speed on the granulometry was carried out for the selected CNT powder, in order to find out if there is a preferred speed range.

As in all references identified, a test tube was chosen as recipient.

Then, a particular effort was conducted with regard to characterization of the aerosols. Measurements by a set 
of "aerosol" measurement devices relevant for CNTs were carried out and a particular effort was made to obtain information on the mass concentration, firstly via an integrated sample and quantification of the carbon and secondly via the use of a microbalance for measurement in real time.

The ultimate objective of the study was to provide recommendations and prospects with regard to the use of the vortex shaker method for the aerosolization of carbon nanotubes, in view of firstly tests on the dustiness of nanopowders and secondly the production of atmospheres enabling the realisation of toxicological studies.

\section{Material and Methods}

For safety purposes, all equipment is placed in a confined facility $[17,18]$ in order to prevent the person conducting the experiment from being accidentally exposed to aerosols [19].

\subsection{Materials}

2.1.1. Material Characterization. The product used in this study was multiwalled carbon nanotubes "Graphistrength C100," hereafter called "C100" [20] produced by the company ARKEMA.

The general characteristics of the C100, determined by the manufacturer, are summarised in Table 1.

An analysis of the granulometry encountered upon manufacture of the $\mathrm{C} 100$ was conducted by the manufacturer and is summarised in Table 2. This analysis was conducted by laser diffraction with a Malvern Mastersizer S granulometer, a low quantity of powder having been placed in solvent. This technique measures the volume of the particles and estimates the volume percent of the particles as a function of the size. It should be noted that the results obtained are based on the characterization of 18 samples corresponding to different batches of $\mathrm{C} 100$.

The data thus obtained highlight a granulometry centred around $400 \mu \mathrm{m}$, with fragments of pellets of less than $15 \mu \mathrm{m}$ representing a volume of under $0.23 \%$. Consequently, only a minority of the volume of particles corresponded to inhalable particles.

On the other hand, the implementation of ultrasound over these samples greatly modifies the granulometry: the average diameter drops to around $40 \mu \mathrm{m}$, which is a fall of a factor of 10. Furthermore, the share of the particles less than $15 \mu \mathrm{m}$ increases greatly to reach around $13 \%$. This significant impact of the ultrasounds highlights a potential for dispersion and/or mechanical rupture of this powder.

C100 potentially contains impurities such as $\mathrm{Fe}$ or $\mathrm{Al}$.

2.1.2. Material Preparation. A volume of $2 \mathrm{~cm}^{3}$ powder was used for each test. This volume was determined using a calibrated crucible of $1 \mathrm{~cm}^{3}$.

In order to determine the repeatability of this operation, the test tube was weighed before and after its filling in 8 cases. The CNT mass was deducted: it is $0.197 \mathrm{~g}$ and presents
TABLE 1: General physical and mechanical properties of this material.

\begin{tabular}{lc}
\hline Parameter & Value \\
\hline Structure & Coils of graphite planes \\
Specific gravity & $2.0 \mathrm{~g} / \mathrm{cm}^{3}$ \\
Apparent density & $0.15 \mathrm{~g} / \mathrm{cm}^{3}$ \\
Specific surface & $100-250 \mathrm{~m}^{2} / \mathrm{g}$ \\
Color & Black \\
Electrical behavior & Metallic \\
Mechanical behaviour & Youngs modulus +1 Tpa \\
Thermal behavior & $>3,000 \mathrm{~W} / \mathrm{m}-\mathrm{K}$ (exceeds \\
\end{tabular}

a standard deviation of $0.005 \mathrm{~g}$, which is a relatively modest standard deviation $(2.5 \%)$

These weighings make it possible to establish that the apparent volume mass of the CNT powder is around $0.1 \mathrm{~g} / \mathrm{cm}^{3}$. This result is consistent with the manufacturer's data (cf. Table 1) indicating a value of $0.15 \mathrm{~g} / \mathrm{cm}^{3}$. These values are comparable to the orders of magnitude encountered by Ogura et al. [3] in a study relating to 6 types of MWCNT, with the range being from around 0.01 to $0.29 \mathrm{~g} / \mathrm{cm}^{3}$.

2.2. Aerosolization Device. The suspension by vortex shaker system is comparable to that used previously in a study on aerosolization on fine alumina [9]. This previous study, in the particular case of the powder it considered, highlighted that aerosolization can only be obtained above a minimum speed of agitation of $1000 \mathrm{rpm}$. It also showed the existence of two zones of interest: firstly the "intermediate speed level" zone, from around 1000 to $1800 \mathrm{rpm}$, and secondly, for higher speeds, the "high speed level" zone, which stands out owing to significant transformation of the powder. This transformation must be avoided in view of the objectives of this study; this is why it concentrates on a range of rotational speed from 1000 to $2000 \mathrm{rpm}$, in order to identify a zone comparable to the "intermediate speed level" zone observed in [9].

The supply speed of the test tube was indicated in the previous study $(2 \mathrm{~L} / \mathrm{min})$. On the other hand, in order to adapt to an instrumentation specific to CNT, the dilution flow rate was increased from $6 \mathrm{~L} / \mathrm{min}$ to $8 \mathrm{~L} / \mathrm{min}$ in the case presented here. This flow rate is set and achieved by the pumps internal to the downstream measurement devices, described below (see Section 2.3.2). The tubes used here are "Conductive Silicone Tubing for Particle Transport" provided by TSI (TSI Inc., St. Paul, Minnesota, USA) with an 0.19 inch inside diameter. Their lengths were kept as short as possible but, however, represent a total length of more than $120 \mathrm{~cm}$ between the aerosol source and measurement device.

The tests mainly used $60 \mathrm{~mL}$ test tubes identical to the previous study; they were replaced with a $30 \mathrm{~mL}$ tube for the feasibility study of a measurement by microbalance and occasionally within the framework of the study on the influence of the geometry of the system.

Occasionally, a cylindrical "buffer" chamber of $200 \mathrm{cc}$ volume was introduced, made from stainless steel. 
TABLE 2: Analysis of the granulometric data on the production of raw product C100 based on 18 samples of several batches.

\begin{tabular}{lcccccccc}
\hline \multirow{2}{*}{ Method of production } & \multicolumn{3}{c}{ Without ultrasound } & \multicolumn{3}{c}{ With ultrasound } \\
& Average & Std. dev. & Average & Std. dev. & Average & Std. dev. & Average & Std. dev. \\
& 382.02 & 28.83 & 0.23 & 0.22 & 44.81 & 6.24 & 12.63 & 3.53 \\
Continuous & 456.40 & 18.45 & 0.23 & 0.13 & 38.78 & 2.10 & 13.14 & 3.73 \\
Batch & & &
\end{tabular}

\subsection{Characterization of the Aerosol. The instrumentation and} the theoretical concepts specific to aerosol physics apply all the more easily to an aerosol when it is composed of for example homogenous spherical particles, of a density close to 1 .

The aerosol derived from a carbon nanotube powder can be considered extreme in relation to this ideal situation: as this study confirms (cf. Section 3.2), this aerosol is constituted partly of independent or very closely associated fibres of a spherical form; agglomerates/pellets of shape similar to a sphere remain constituted of fibres which gives them a high porosity and consequently a particularly low volume mass, which makes them again atypical. This difficulty is highlighted by [8], which stresses the need for additional studies on the "extreme shape" and the "structure influences" of nanoplates and nanofibres.

This difficulty can be illustrated by the case of measurement by differential mobility analyser (DMA). This technique is one of the most common to date techniques in aerosol metrology and has been placed by [8] on the list of recommended techniques to study dustiness. Ku et al. [21] studied the capacity of a differential mobility analyzer (DMA) to measure ultrafine fibrous carbon aerosols. They highlight that this technique presents "anomalous responses and that measurement of nanometer-diameter conducting fibrous material by electrical mobility analysis may present a unique challenge."

It is therefore established that, in the case of measuring a $\mathrm{CNT}$ aerosol, the selection of measurement devices and the interpretation of data must be conducted with precaution.

In view of these difficulties, the choice was made in this study

(i) firstly to draw on qualitative observations by microscope: the use of microscopy is indeed widespread and recognised in the case of asbestos fibrous particles;

(ii) secondly to seek to implement techniques for quantification not in number but in mass: total sampling on filter is again recognised for asbestos and in many fields such as occupational hygiene.

The characterization of the aerosol was conducted principally using two filtration based collection devices: firstly the miniparticle sampler (MPS) [22] and secondly a total filter coupled with a deferred analysis. A microbalance [23] was also used in part of the study in order to study the feasibility of the time monitoring of emissions via measurement of the mass concentration.
2.3.1. Qualitative Analysis by Microscopy. As granulometers can encounter difficulties owing to the fibrous structure of the aerosol, the study of the distribution in size was carried out through sampling on electronic microscope grid, with this approach also making it possible to study the nanostructured nature of the aerosol.

A sample collector-the miniparticle sampler (MPS) allows extracting particle samples on a TEM grid from the aerosol [22]. The air sample was taken via a Gilian LFS-113DC pump, on Quantifoil copper-carbon grids (Oxford).

The samples have been investigated with respect to their particle shapes and sizes by means of a transmission electron microscope (TEM, FEI CM12, operated at $120 \mathrm{kV}$ ).

2.3.2. Quantitative Analysis. The quantification was conducted by sampling on total filter and analysis of total carbon.

Different filter holders were used in preliminary tests. It appeared quite quickly that the sample of MWCNT C100 was very sensitive to electrostatic effects, which can entail losses of matter on the filter holder and also on the joint. Consequently, filter, filter holder, and joint were selected so as to limit the electrostatic effects (metal filter holder) and limit losses; the joint used is of the same material as the filter (quartz), which makes it possible to incinerate it rather than recover the CNT deposits from its surface.

Samples of one hour were taken at a speed of $5 \mathrm{~L} / \mathrm{min}$ on $25 \mathrm{~mm}$ quartz filters placed within metal filter holders (Merck-Millipore).

The carbon nanotubes deposited on the filter are oxidized by combustion at $980^{\circ} \mathrm{C}$ in the module SSM $5000 \mathrm{~A}$ (solid sample module). The melting point is lowered by addition of $\mathrm{V}_{2} \mathrm{O}_{5}$. Platinum is used as catalyzer. The carbon is then released in $\mathrm{CO}_{2}$ measured by nondispersive infrared analysis by a Shimadzu detector. To lower the quantification limit, a second infrared detector, the MIR6000, was added in series to the first one. This assembly allows obtaining a limit of quantification of $5 \mu \mathrm{g} /$ filter.

The presence of the elements $\mathrm{Al}$ and Fe was also quantified by optical inductively coupled plasma (ICP-AES) and by inductively coupled plasma mass spectrometry (ICP-MS).

Each test was repeated a minimum of 3 times. The dispersion over the results remains significant with a relative standard deviation of a minimum of $30 \%$. Improvement efforts could be made in respect of sampling in order to reduce its contribution to this dispersion.

2.3.3. Monitoring over Time. The monitoring in real time of the mass concentration of an aerosol can be addressed via indirect or direct measurements. 
The indirect measurement can be taken with different types of granulometers (optical, DMA, etc.) supplying a granulometry in number. To do this, it is necessary to compare the real particles to model particles, in this instance spherical, and known volume mass. This approach is particularly tricky as we have seen in the case of a fibrous aerosol.

Direct measurements implement physics principles in direct connection with the mass concentration. The tapered element oscillating microbalance (TEOM-series 1400A, Thermo Electron Corporation, USA) is one of the rare possibilities in this field. In this instance, this technique assesses particle mass concentration from the mass-dependent frequency changes of an oscillating filter [23].

The fibrous nature of the CNT aerosol does not as a rule raise any difficulty for the measurement cell of this technique. Indeed, the collection is assured by a total filter and the measuring-based on a vibratory principle-only depends on the mass of matter collected.

However, the microbalance presents a certain number of practical limits, such as its volume and its form (vertical). It is also deemed to require a certain amount of time to stabilise upon start-up or in the presence of significant changes to properties of the air, such as pressure or humidity. In this case, humidity did not pose any difficulty as the supply of the vortex agitator and the dilution systems was assured with dry air.

\section{Results}

3.1. Study of the Feasibility of Real Time Measurement by Microbalance. The objective of this part of the works relates to the study of the feasibility of the implementation of a TEOM microbalance to measure the mass concentration of the aerosol in real time.

The implementation of the microbalance within the circuit straight away raised a problem: it proved very difficult to stabilise the signal and consequently to validate the data. The hypotheses have been raised that this instability was either due to the variations in pressure induced by the agitation of the powder by the vortex shaker or due to the vibration in air flow by the pumps of the downstream measurement devices, which, however, should be electronically stabilized. The solution envisaged and implemented consists of the introduction of a "buffer" chamber (cf. Figure 1) upstream of the microbalance, the objective of which is to attenuate the fluctuations in pressure by passing the aerosol into a volume kept constantly at atmospheric pressure. To do this, a high efficiency particulate air (HEPA) filter is connected firstly to this chamber and secondly to the nanosecured facility. This filter is of large section in order to facilitate the balancing process. Average time monitoring was thus obtained (Figure 2) on the basis of 4 tests carried out at $1500 \mathrm{rpm}$ with a $30 \mathrm{~mL}$ tube. These results show that the implementation of this "buffer" chamber effectively enabled the microbalance to provide a stable signal.

However, different aspects must be considered: (1) as will be shown in Section 3.4 (influence of the geometry), the introduction of the buffer chamber entails a reduction in

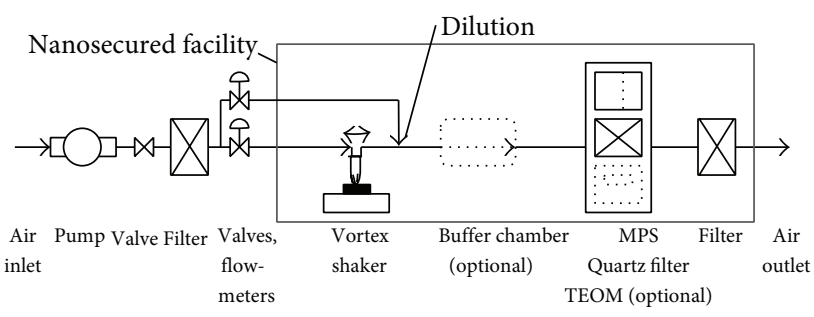

FIGURE 1: The experimental setup. The components used occasionally (buffer chamber, TEOM) are represented in dotted lines.

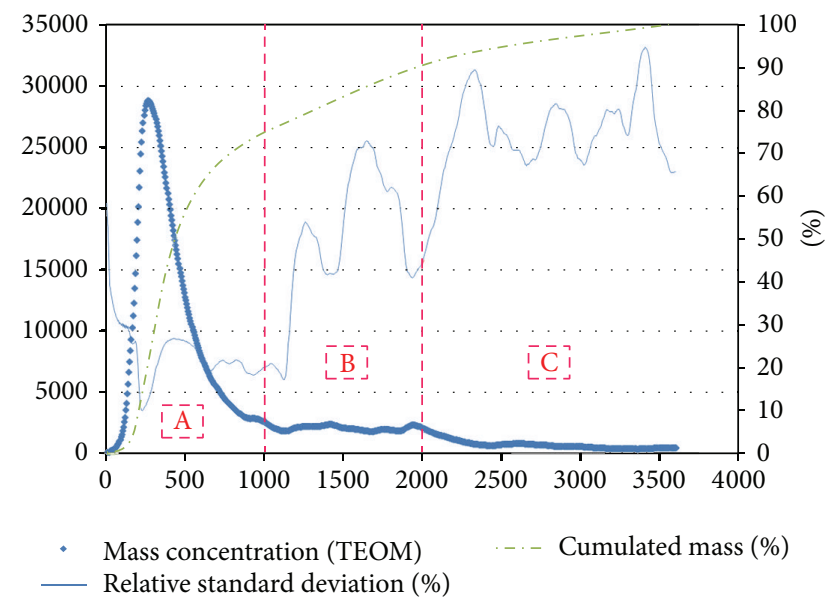

FIgURE 2: Monitoring over time (s) of the mass concentration of the aerosol by microbalance $\left(\mu \mathrm{g} / \mathrm{m}^{3}\right) ; 30 \mathrm{~mL}$ tube, $1500 \mathrm{rpm}$, average over 4 tests.

concentrations, probably owing to aerosol deposits within it; (2) total carbon measurements have been taken simultaneously (Table 3). The sampling on filter is generally considered a reference: this is the case, for example, in the field of surveillance of air quality in ambient air (cf. standard EN 12341). If we apply this principle, the results will show values reduced by a factor of around 2.5 , that is, an underestimate of $60 \%$ of the microbalance in comparison to the carbon analysed on the total filter. Various explanations could be put forward, including a risk of deposit in the TEOM, particularly in the vertical tube placed upstream of the balance or the fact that the quartz fiber filters for total carbon measurement could adsorb condensable gaseous organic carbon (OC), pumped from the outside air or evaporated from the pump, connecters, and tubing. The carbon analysis used in this study cannot differentiate between elemental carbon (such as CNTs) and OC collected/adsorbed on quartz fiber filters. Significant work would therefore need to be conducted to analyse the situation and improve performances. Consequently, in view of these limitations, the results of the TEOM measurement presented here must be considered only as indicatory values.

\subsection{Characterization of the Aerosol Generated at $1500 \mathrm{rpm}$.} The results of the characterization of the aerosol obtained by agitation of a $60 \mathrm{~mL}$ tube at a speed of $1500 \mathrm{rpm}$ are presented in this section. 
TABLE 3: Comparison of the mass collected by the microbalance with the carbon mass on the total filter. 4 tests.

\begin{tabular}{lcccccr}
\hline & \multicolumn{3}{c}{ Mass $(\mu \mathrm{g})$} & Average & Standard deviation \\
\hline TEOM & 1001 & 1159 & 1211 & 1133 & & \\
Total Filter (Carbon) & 4405 & 2882 & 3149 & 1862 & 0.4 & 0.2 \\
RATIO: TEOM/Total Filter & 0.2 & 0.4 & 0.4 & 0.6 & 0.4 \\
\hline
\end{tabular}

TABLE 4: Aerosolized particle mass ( $\mu \mathrm{g}) /$ filter for different parameterizations (rotational speed, geometry.). The mass concentration $\left(\mathrm{mg} / \mathrm{m}^{3}\right.$ ) can be obtained by dividing the masses in $\mu$ g by 300 . " $n$ " indicates the number of tests.

\begin{tabular}{|c|c|c|c|c|c|c|c|c|c|c|c|c|}
\hline \multirow{3}{*}{$\begin{array}{l}\text { Rotational } \\
\text { speed (rpm) }\end{array}$} & \multicolumn{8}{|c|}{$60 \mathrm{~mL}$ tube } & \multirow{2}{*}{\multicolumn{4}{|c|}{$\begin{array}{l}30 \mathrm{~mL} \text { tube } \\
\text { Without chamber }\end{array}$}} \\
\hline & \multicolumn{4}{|c|}{ Without chamber } & \multicolumn{4}{|c|}{ With chamber } & & & & \\
\hline & $\mu \mathrm{g}$ & $n \#$ & $\begin{array}{c}\text { Average } \\
\mu \mathrm{g}\end{array}$ & $\begin{array}{c}\text { Rel. Std. } \\
\text { Dev. } \\
\%\end{array}$ & $\mu \mathrm{g}$ & $n \#$ & $\begin{array}{c}\text { Average } \\
\mu \mathrm{g}\end{array}$ & $\begin{array}{c}\text { Rel. Std. } \\
\text { Dev. } \\
\%\end{array}$ & $\mu \mathrm{g}$ & $n \#$ & $\begin{array}{c}\text { Average } \\
\mu \mathrm{g}\end{array}$ & $\begin{array}{c}\text { Rel. Std. } \\
\text { Dev. } \\
\%\end{array}$ \\
\hline 1000 & $\begin{array}{l}148 \\
361 \\
469 \\
296 \\
\end{array}$ & 4 & 319 & $42 \%$ & & & & & & & & \\
\hline 1250 & 823 & 1 & 823 & l & $\begin{array}{c}278 \\
480 \\
78 \\
\end{array}$ & 3 & 279 & $72 \%$ & & & & \\
\hline 1500 & $\begin{array}{c}1723 \\
973 \\
1128 \\
868\end{array}$ & 4 & 1173 & $33 \%$ & & & & & $\begin{array}{l}2680 \\
4405 \\
2882 \\
3149 \\
1862 \\
\end{array}$ & 5 & 2996 & $31 \%$ \\
\hline 1750 & & & & & $\begin{array}{l}389 \\
765 \\
553 \\
\end{array}$ & 3 & 569 & $33 \%$ & & & & \\
\hline 2000 & $\begin{array}{l}1282 \\
1702 \\
2602 \\
\end{array}$ & 3 & 1862 & $36 \%$ & & & & & & & & \\
\hline
\end{tabular}

The average quantity of carbon sampled during 4 tests of one hour on the total filter (cf. Table 4) is $1.2 \mathrm{mg}$, with the relative standard deviation being $33 \%$. This quantity corresponds to around $6 \%$ of the initial mass of powder.

The manufacturer indicates that the production of MWCNT by CCVD (catalytic chemical vapor deposition) entails the presence of iron and aluminium in the powder, at a level of a few percent in mass. R'mili et al. [24] showed that these elements are also found in the aerosol derived from this MWCNT powder. In this study, to verify this characteristic, these constituents were quantified for guideline purposes for a test: the results confirm their presence in the aerosol at the level of around $6 \%$ of the mass of the sample, which is comparable to the order of magnitude announced by the manufacturer for the powder itself.

The distribution in size of the aerosol and the shape and elementary composition of the particles were studied from a qualitative point of view by microscopy (cf. Figure 3 ). The particles observed can be put into 4 categories:

(i) pellets (cf. Figure 3(a)): these fibrous particles present a diameter of at least $5 \mu \mathrm{m}$;

(ii) fragments of clumps of 1 to $2 \mu \mathrm{m}$ (cf. Figure $3(\mathrm{~b})$ ); (iii) small agglomerates composed of a few nanotubes (between 3 and 10 nanotubes) (cf. Figures 3(c) and 3(d));

(iv) isolated nanotubes (cf. Figures 3(e) and 3(f)).

It should be noted that these results, found by microscopic observations, are comparable to those obtained on site by R'mili et al. [24].

These results show that all particles generated upon aerosolization are fibrous, with the arrangements varying from the pellet to the isolated fibre. This confirms the importance that has been given to the fibrous nature of the aerosol in the choice of instrumentation selected for this study (cf. Section 2.3).

While keeping in mind the preliminary and strictly indicatory nature of the observations made by TEOM microbalance (cf. Section 3.1), it is possible to benefit from the average time monitoring of the TEOM derived from 4 tests conducted with a $30 \mathrm{~mL}$ tube. This (Figure 2 ) is mainly characterized by a substantial concentration peak at the start of the test (zone "A," from 0 to $17 \mathrm{~min}$ ), giving way to a first plateau (zone "B," from the 17 th to the 34 th minute) and then to a second plateau (zone "C"), both of lesser concentration. The appearance of a peak at the start of the test is consistent with the results 


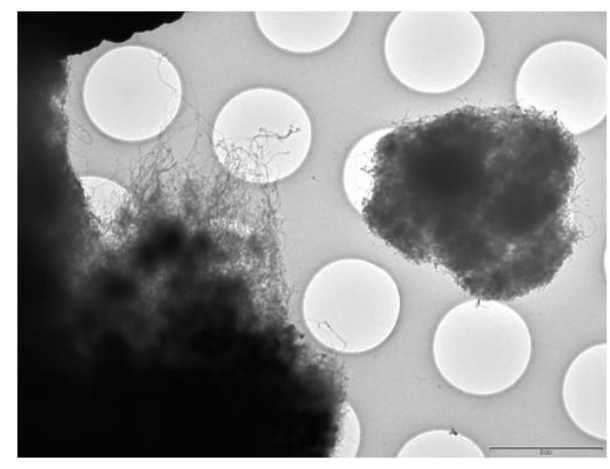

(a) Pellet of $5 \mu \mathrm{m}$ (scale $2 \mu \mathrm{m})$

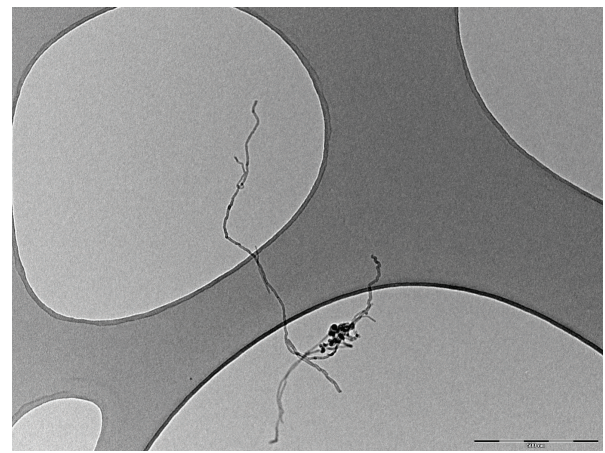

(c) Agglomerates of several fibres (scale $200 \mathrm{~nm}$ )

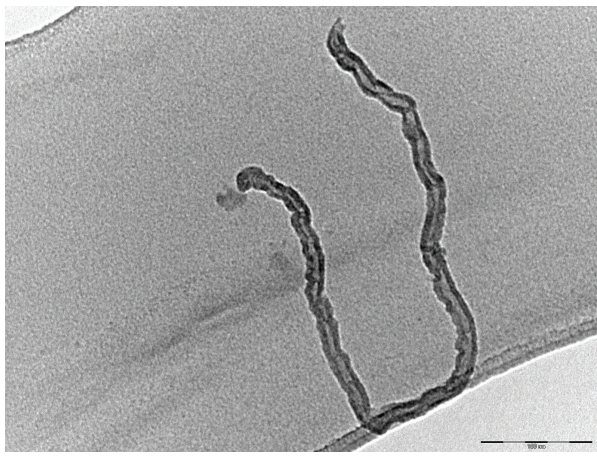

(e) Isolated carbon nanotubes (scale $100 \mathrm{~nm}$ )

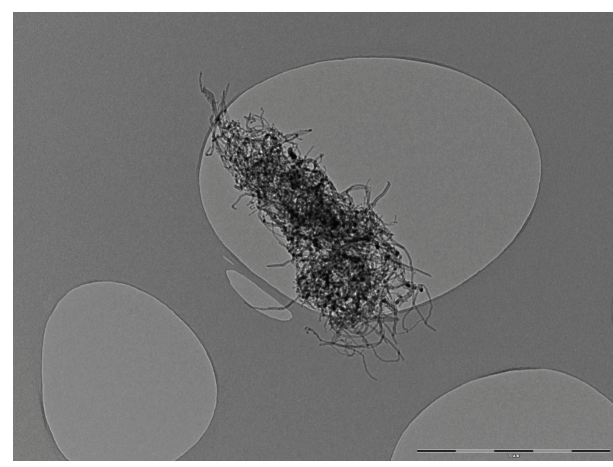

(b) Fragments of pellet of 1 to $2 \mu \mathrm{m}$ in length (scale $1 \mu \mathrm{m})$

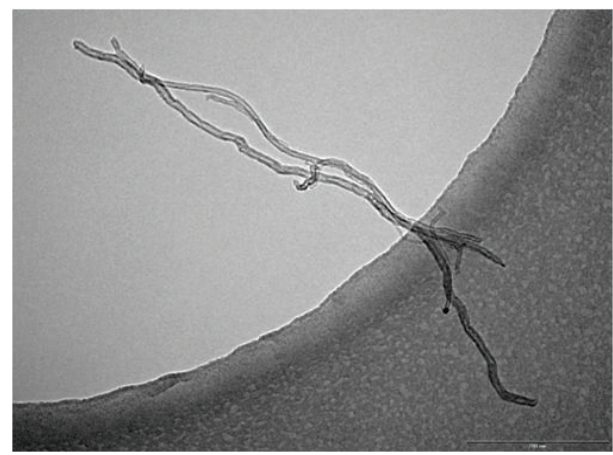

(d) Agglomerates of several fibres (scale $200 \mathrm{~nm}$ )

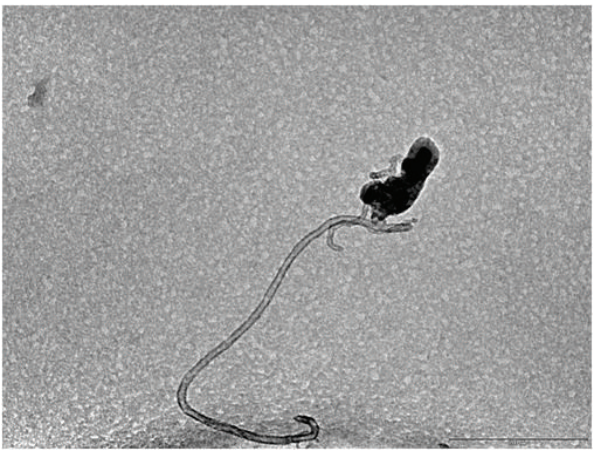

(f) Isolated carbon nanotubes (scale $100 \mathrm{~nm}$ )

FIGURE 3: TEM pictures of aerosolized particles (1500 rpm).

obtained elsewhere by Morgeneyer et al. [9] in the case of an alumina powder: this seems to confirm the fact that starting the vortex shaker at the agitation speed of use (here $1500 \mathrm{rpm}$ ) causes a very high emission. This phase is temporary since the system then moves on to a stabilised emission phase, at a lesser concentration level. On the other hand, in the case of the MWCNT GC100, the share of matter emitted in this start-up peak is particularly high: the concentration during the peak is around 10 times higher than the concentration of the first plateau, with this factor only being 3 in the case of alumina [9]; more specifically, the time monitoring of the cumulative mass (cf. Figure 2) shows that the initial peak represents $75 \%$ of the aerosolized mass throughout the test. These results must be taken into account, particularly in the case of development of toxicology tests for which a stable concentration is sought over time.
3.3. Influence of the Rotational Speed. The influence of the rotational speed on the aerosolization behaviour was studied in the range from 1000 to $2000 \mathrm{rpm}$. The emissivity was determined via the quantity of carbon aerosolized.

The results obtained (cf. Table 4 and Figure 4) show that the increase in speed entails an increase in the aerosol mass produced, which is consistent with the results of Morgeneyer et al. [9] obtained for an alumina powder. On the other hand, the growth is much more modest here: the switch from a speed of 1000 to $2000 \mathrm{rpm}$ only increases the emission by a factor of 6, against a factor of around 1500 in the case of Morgeneyer et al. [9].

In the case studied here, the emission/speed relationship can be modelled by a linear function (Figure 4).

Semiquantitative analyses by microscopy were also conducted. They highlight that the increase in speed entails an 


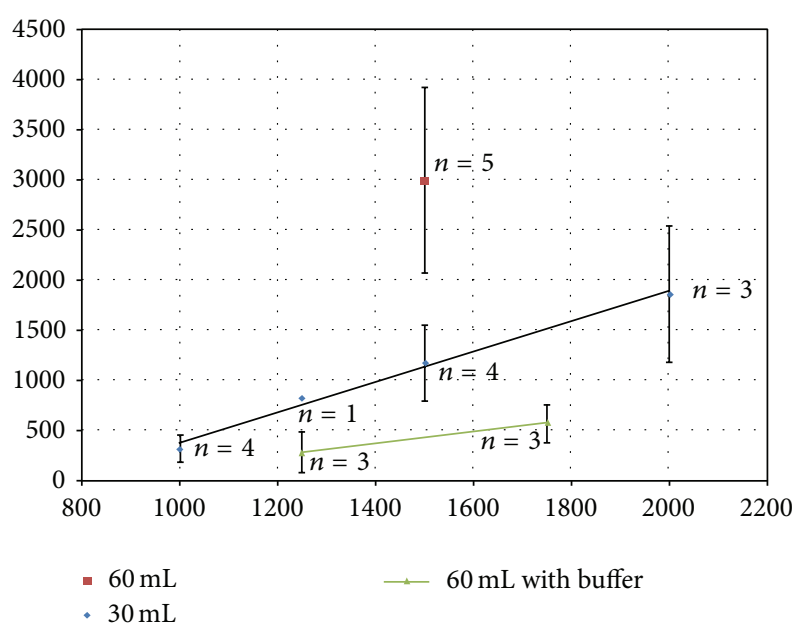

FIGURE 4: Mass of aerosol/filter as a function of the rotational speed and of the geometry of the system.

increase in the proportion of isolated fibres. This observation suggests that the increase in speed encourages a phenomenon of deagglomeration of the powder. In this instance, we can assume that getting the powder moving entails its elementsparticularly the pellets-rubbing against each other, causing breakage, tearing, and consequently a reduction in the granulometry of the powder, which entails an increase in the number of particles able to be suspended. There are elements of information to strengthen this hypothesis: firstly, the analyses conducted by the manufacturer (cf. Section 2.1.1) have shown the capacity of the MWCNT C100 powder to disperse; secondly, Morgeneyer et al. [9] observed a similar phenomenon with an alumina powder; lastly, this alumina powder is composed of solid integrated elements, generally less fragile than the fibrous pellets composing the MWCNT C100 powder.

In conclusion, while the increase in speed of agitation makes it possible to increase the mass of aerosolized particles per time unit, it entails modifications to the granulometry of the aerosol with an increase in the proportion of isolated fibres. This aspect must be taken into account, notably in the discussion on the representativeness of the system according to the type of application.

3.4. Influence of the Geometry of the System. The influence of the geometry of the system was addressed in two ways: firstly by introducing an additional module to the reliant shaker and instrumentation line and secondly by modifying the volume of the test tube (comparison between a volume of $30 \mathrm{~mL}$ and $60 \mathrm{~mL}$ ).

The results are presented in Table 4 and Figure 4.

The introduction of an additional module-in this instance a buffer chamber-causes a significant reduction in the quantity of matter reaching the measuring devices. The reasons for this may be firstly that the introduction of this system increases the duration of transport between vortex shaker and instrumentation, which leaves more time for the deposit processes to act (e.g., by sedimentation); secondly the geometry of the buffer chamber has not been evaluated with regard to deposits, and its form may favour certain deposit phenomena (e.g., losses by diffusion and impaction).

Conversely, the use of a test tube of a lesser volume entails a significant increase in the emission. Thus the aerosolized matter increases from $6 \%$ to $19 \%$ of the bulk mass, respectively, for a tube of 60 and $30 \mathrm{~mL}$.

These tests clearly demonstrate the influence of the geometry of the system. Consequently, this means that the comparison of results within a laboratory and, beyond, between laboratories, suggests a strict similarity of devices and parameters of operation.

\section{Discussion}

This section has the objective of discussing the results obtained in this study, given the needs firstly in the matter of dustiness (Section 4.1) and secondly in the matter of development of toxicology tests (Section 4.2). Perspectives are proposed.

4.1. Discussion in View of the "Dustiness" Specifications. The aerosolization caused by the system and the protocol as things stand is characterized by an emission that concentrates in the first quarter hour of experimentation.

In terms of representativeness, this relatively short timescale could suit for dustiness tests because many work phases encountered in the field are relatively short: bagging, transport, and so forth. Furthermore, it could be considered that these phenomena are mostly caused suddenly, which is also the case of the protocol used for the vortex (upon startup, immediate switch to the target agitation speed).

If we put the real time monitoring to one side and concentrate on the sampling on filter integrating the whole of a test, we can consider that the device is in the operational condition to study the field situations for which it will be deemed representative. A simple improvement could be made by integrating a regulatory cut-off upstream of the total filter (Table 5).

These conclusions and the results presented elsewhere seem to orientate the protocol towards a test duration of around 15 minutes, for a speed of agitation of $1500 \mathrm{rpm}$.

4.2. Discussion in View of the "Toxicology" Specifications. According to the assessment drawn up in Table 6, improvements must be made with regard to stability and repeatability if we want the vortex shaker applied to the Graphistrength C100 to become a tool operating routinely.

Indeed, the initial peak represents too great a share of the quantity of aerosolized matter: a rebalance must be sought to the benefit of the two subsequent plateaus. Once this objective is reached, it would also be necessary to extend the duration of the tests from one to several hours.

The results obtained by Fujitani et al. [6] on a Siever system suggest that the use of bronze beads would facilitate obtaining a stable emission. However, the use of these beads firstly may present a risk of interference (creation of an aerosol, for example, upon friction between beads), but 
TABle 5: Dustiness-objectives, contribution of this study and perspectives. Level of effort: moderate, significant, very significant.

\begin{tabular}{|c|c|c|}
\hline Objectives (cf.1) & Contribution of this study & Perspectives \\
\hline Representativeness & $\begin{array}{l}\text { The study of time monitoring of the mass concentration } \\
\text { was instigated. The emission of matter seems to be } \\
\text { concentrated over the first } 152 \text { (10) minutes. } \\
\text { These characteristics orientate the representativeness } \\
\text { towards short processes of a few minutes. } \\
\text { The transformation of the powder observed upon } \\
\text { increase in speed favours remaining within a moderate } \\
\text { speed ( } 1500 \mathrm{rpm}) \text {. }\end{array}$ & $\begin{array}{l}\text { Identify processes best corresponding to } \\
\text { these characteristics, and implement the } \\
\text { device as it is. }\end{array}$ \\
\hline Mesurands & $\begin{array}{l}\text { Implementation of total sampling (without size } \\
\text { selection) and analysis of the total carbon. } \\
\text { Real time monitoring: feasibility of measurement by } \\
\text { microbalance. }\end{array}$ & $\begin{array}{l}\text { Introduction of samplers on filter with } \\
\text { regulatory cut-off (inhalable, thoracic, } \\
\text { alveolar). } \\
\text { Continue integration of the microbalance. } \\
\text { Implement a simple indicator in parallel } \\
\text { (COP or carbon monitoring). }\end{array}$ \\
\hline Easy-to-use & $\begin{array}{l}\text { The device implies its implementation at a secure } \\
\text { station. } \\
\text { A relatively simple protocol has been implemented. }\end{array}$ & $\begin{array}{l}\text { Creation of a pre-normative protocol, } \\
\text { drawing on existing standards. }\end{array}$ \\
\hline
\end{tabular}

TABLE 6: Toxicology-objectives, contribution of this study and perspectives.

\begin{tabular}{|c|c|c|}
\hline Objectives (cf.1) & Contribution of this study & Perspectives \\
\hline Stability & $\begin{array}{l}\text { The study of time monitoring of the mass concentration } \\
\text { was instigated. The emission of matter seems to be } \\
\text { concentrated over the first } 15 \text { (10) minutes. }\end{array}$ & $\begin{array}{l}\text { The device must progress to achieve stability } \\
\text { over several hours. }\end{array}$ \\
\hline Repeatability & $\begin{array}{l}\text { Establishment of the stability is a prerequisite. } \\
\text { The standard deviation over the total carbon } \\
\text { concentration was quantified. }\end{array}$ & $\begin{array}{l}\text { It is necessary to seek to reduce the } \\
\text { dispersion of results. The effort must relate } \\
\text { to improving the total sampler, but also the } \\
\text { suspension system. }\end{array}$ \\
\hline Representativeness & $\begin{array}{l}\text { The transformation of the powder observed upon } \\
\text { increase in speed favours remaining within a moderate } \\
\text { speed ( } 1500 \mathrm{rpm}) \text {. On the other hand, the stability is not } \\
\text { established over time: discussion on the } \\
\text { representativeness is therefore premature. }\end{array}$ & $\begin{array}{l}\text { Generation must firstly be stabilised over } \\
\text { time. }\end{array}$ \\
\hline $\begin{array}{l}\text { Choice of } \\
\text { concentration }\end{array}$ & $\begin{array}{l}\text { The influence of the geometry of the system has been } \\
\text { demonstrated. Adjustment parameters are available: } \\
\text { quantity of powder used, size of the test tube. }\end{array}$ & $\begin{array}{l}\text { Once the generation is stabilised, these } \\
\text { parameters will enable adjustment of the } \\
\text { level of concentration. This adjustment will } \\
\text { probably be specific to each type of powder. }\end{array}$ \\
\hline Mesurands & Cf. Table 5 & \\
\hline Interferences & $\begin{array}{l}\text { The presence of } \mathrm{Fe} \text { and } \mathrm{Al} \text { is confirmed. These do not } \\
\text { interfere as they are present in the initial powder. }\end{array}$ & $\begin{array}{l}\text { There is no particular worry on this point } \\
\text { but it must be verified by a broad analysis of } \\
\text { the components of the aerosol. }\end{array}$ \\
\hline
\end{tabular}

particularly secondly it will entail a break with the field situation (representativeness).

The results obtained confirm a trend observed for alumina [9], namely, the impact of high speeds of agitation on the granulometry of the aerosol. Consequently, the moderate speed zone (1500 rpm) must be recommended, particularly for this application since it targets durations of operation of several hours.

\section{Summary and Conclusion}

The growing use of nanomaterials entails emerging risks for humans and the environment. Taking these risks into account requires the development of new tools. The study presented here falls within this approach: it aims to evaluate and develop the vortex shaker technique as tool to determine the dustiness of a powder and as means of generating atmosphere in experimental toxicology. All of this work related to the Graphistrength C100 manufactured by ARKEMA.

The concept of "representativeness" was placed at the heart of this work. The objective of this concept was to select and configure experimental devices (laboratory) as close as possible to the true conditions of exposure. This approach explains the nonuse of beads in the test tube; indeed, while these beads facilitate the practical realisation of the tests, they remove the device from the conditions encountered in the field. 
The mass concentration is a metric given preference by the regulatory texts in industrial hygiene. This is why one objective of this study was to study the feasibility of measuring the mass concentration in real time via a microbalance. The feasibility was demonstrated, even though there is still significant work to be done for this microbalance to be operational upon leaving the vortex shaker. The time monitoring thus produced played an important role in the interpretation of the study data, which justifies continuation of this area of work.

The aerosol derived from the agitation of Graphistrength $\mathrm{C} 100$ at $1500 \mathrm{rpm}$ was characterized (total carbon, TEM). Four categories of inhalable particles were observed, being pellets, fragments of pellets, small agglomerates of nanotubes, and lastly isolated nanotubes. These particles are all fibrous and in this instance nanostructured. Aluminium and ironresidues of catalysis-were observed in the aerosol, at a level comparable to that of the powder itself.

This study demonstrates that the speed of agitation and the geometry of the system are influential parameters.

The increase in speed entails an increase in the aerosol mass produced, which is consistent with a previous study [9]. On the other hand, in the case of the Graphistrength C100, the relationship between these two parameters is different, in this instance linear. This result reinforces the idea, already addressed previously [9], that each powder is a specific case, with its own properties and consequently a specific behaviour.

A significant evolution to the granulometry was observed upon increase in speed; to limit this phenomenon, it is recommended to remain between around 1000 and $1700 \mathrm{rpm}$.

The variation in the geometry of the system can cause either a fall or an increase in the mass concentration measured. This property presents a potential for adjustment of the concentration. It is also a key teaching because it requires the implementation of strict common protocols and tools for any community wishing to ensure the comparability of its results.

\section{Outlook}

The results of this study, centred on the C100, were discussed in respect of the needs identified with regard to dustiness and atmosphere generation devices.

It thus appears that the instrumentation must be improved in order to reduce its contribution to the dispersion of the results. Real time measuring of the mass concentration must be developed to achieve operational measuring.

Given a clear argument over the issue of representativeness, the device could as things stand be exploited to characterize the dustiness of situations encountered in industry.

The case of application in toxicology is less mature: time monitoring showed a lack of stability particularly in the startup phase. The development work must be continued, notably to obtain a stable and adjustable concentration.

\section{Conflict of Interests}

The authors declare that there is no conflict of interests regarding the publication of this paper.

\section{Acknowledgments}

The authors would like to thank ARKEMA company (GENESIS Programme) for financing this study, as well as Emmanuel Peyret and Nicolas Chatelier for their technical support.

\section{References}

[1] ISO TS, 80004-1, "Nanotechnologies-Vocabulary-Part 1: Core Terms," 2010.

[2] EN 15051, "Workplace atmospheres-measurement of the dustiness of bulk materials-requirements and choice of reference test methods," 2006.

[3] I. Ogura, M. Kotake, H. Sakurai, and M. Gamo, "Emission and exposure assessment of manufactured nanomaterials," NEDO Project P06041, 2012.

[4] P. T. O'Shaughnessy, M. Kang, and D. Ellickson, "A novel device for measuring respirable dustiness using low-mass powder samples," Journal of Occupational and Environmental Hygiene, vol. 9, no. 3, pp. 129-139, 2012.

[5] P. A. Baron, G. J. Deye, B. T. Chen, D. E. Schwegler-Berry, A. A. Shvedova, and V. Castranova, "Aerosolization of singlewalled carbon nanotubes for an inhalation study," Inhalation Toxicology, vol. 20, no. 8, pp. 751-760, 2008.

[6] Y. Fujitani, A. Furuyama, and S. Hirano, "Generation of airborne multi-walled carbon nanotubes for inhalation studies," Aerosol Science and Technology, vol. 43, no. 9, pp. 881-890, 2009.

[7] J. Pauluhn, "Subchronic 13-week inhalation exposure of rats to multiwalled carbon nanotubes: toxic effects are determined by density of agglomerate structures, not fibrillar structures," Toxicological Sciences, vol. 113, no. 1, pp. 226-242, 2010.

[8] ISO/TS 12025, Nanomaterials-Quantification of Nano-Object Release from Powders by Generation of Aerosols, 2012.

[9] M. Morgeneyer, O. Le Bihan, A. Ustache, and O. AguerreChariol, "Experimental study of the aerosolization of fine alumina particles from bulk by a vortex shaker," Powder Technology, vol. 246, pp. 583-589, 2013.

[10] A. H. M. Andreasen, N. Hofman-Bang, and N. H. Rasmussen, "Über das stäubungsvermögen der stoffe," Kolloid-Zeitschrift, vol. 86, no. 1, pp. 70-77, 1939.

[11] B. M. Wright, "A new dust-feed mechanism," Journal of Scientific Instruments, vol. 27, no. 1, pp. 12-15, 1950.

[12] ASTM D547-41, "Test Method for Index of Dustiness of Coal and Coke (Withdrawn 1986)," 1980.

[13] K. A. Jensen, I. K. Koponen, P. A. Clausen, and T. Schneider, "Dustiness behaviour of loose and compacted Bentonite and organoclay powders: what is the difference in exposure risk?" Journal of Nanoparticle Research, vol. 11, no. 1, pp. 133-146, 2009.

[14] D. Barletta and M. Poletto, "Aggregation phenomena in fluidization of cohesive powders assisted by mechanical vibrations," Powder Technology, vol. 225, pp. 93-100, 2012.

[15] T. Myojo, T. Oyabu, K. Nishi et al., "Aerosol generation and measurement of multi-wall carbon nanotubes," Journal of Nanoparticle Research, vol. 11, no. 1, pp. 91-99, 2009. 
[16] A. D. Maynard, P. A. Baron, M. Foley, A. A. Shvedova, E. R. Kisin, and V. Castranova, "Exposure to carbon nanotube material: aerosol release during the handling of unrefined single-walled carbon nanotube material," Journal of Toxicology and Environmental Health A, vol. 67, no. 1, pp. 87-107, 2004.

[17] O. Le Bihan, N. Shandilya, L. Gheerardyn, O. Guillon, E. Dore, and M. Morgeneyer, "Investigation of the release of particles from a nanocoated product," Advances in Nanoparticles, vol. 2, no. 1, pp. 39-44, 2013.

[18] O. Le Bihan, M. Morgeneyer, N. Shandilya, O. Aguerre-Chariol, and C. Bressot, "Emission chambers, a method for nanosafety", in Handbook on Safe Use of Nanomaterials, Chapter 9.2, Elsevier, 2013.

[19] N. Shandilya, O. Le Bihan, and M. Morgeneyer, "A review on the study of the generation of (nano) particles aerosols during the mechanical solicitation of materials," Journal of Nanomaterials, vol. 2014, Article ID 289108, 16 pages, 2014.

[20] S. Bordere, J. M. Corpart, N. E. El Bounia et al., "Industrial production and applications of carbon nanotubes," Arkema, Groupement de Recherches de Lacq, Lacq, France, http://www.graphistrength.com/.

[21] B. K. Ku, A. D. Maynard, P. A. Baron, and G. J. Deye, "Observation and measurement of anomalous responses in a differential mobility analyzer caused by ultrafine fibrous carbon aerosols," Journal of Electrostatics, vol. 65, no. 8, pp. 542-548, 2007.

[22] B. R'mili, O. L. C. Le Bihan, C. Dutouquet, O. Aguerre-Chariol, and E. Frejafon, "Particle sampling by TEM grid filtration," Aerosol Science and Technology, vol. 47, no. 7, pp. 767-775, 2013.

[23] H. Patashnick and E. G. Rupprecht, "Continuous PM-10 measurements using the tapered element oscillating microbalance," Journal of the Air \& Waste Management Association, vol. 41, no. 8, pp. 1079-1083, 1991.

[24] B. R'mili, C. Dutouquet, J. B. Sirven, O. Aguerre-Chariol, and E. Frejafon, "Analysis of particle release using LIBS (laser-induced breakdown spectroscopy) and TEM (transmission electron microscopy) samplers when handling CNT (carbon nanotube) powders," Journal of Nanoparticle Research, vol. 13, no. 2, pp. 563-577, 2011. 

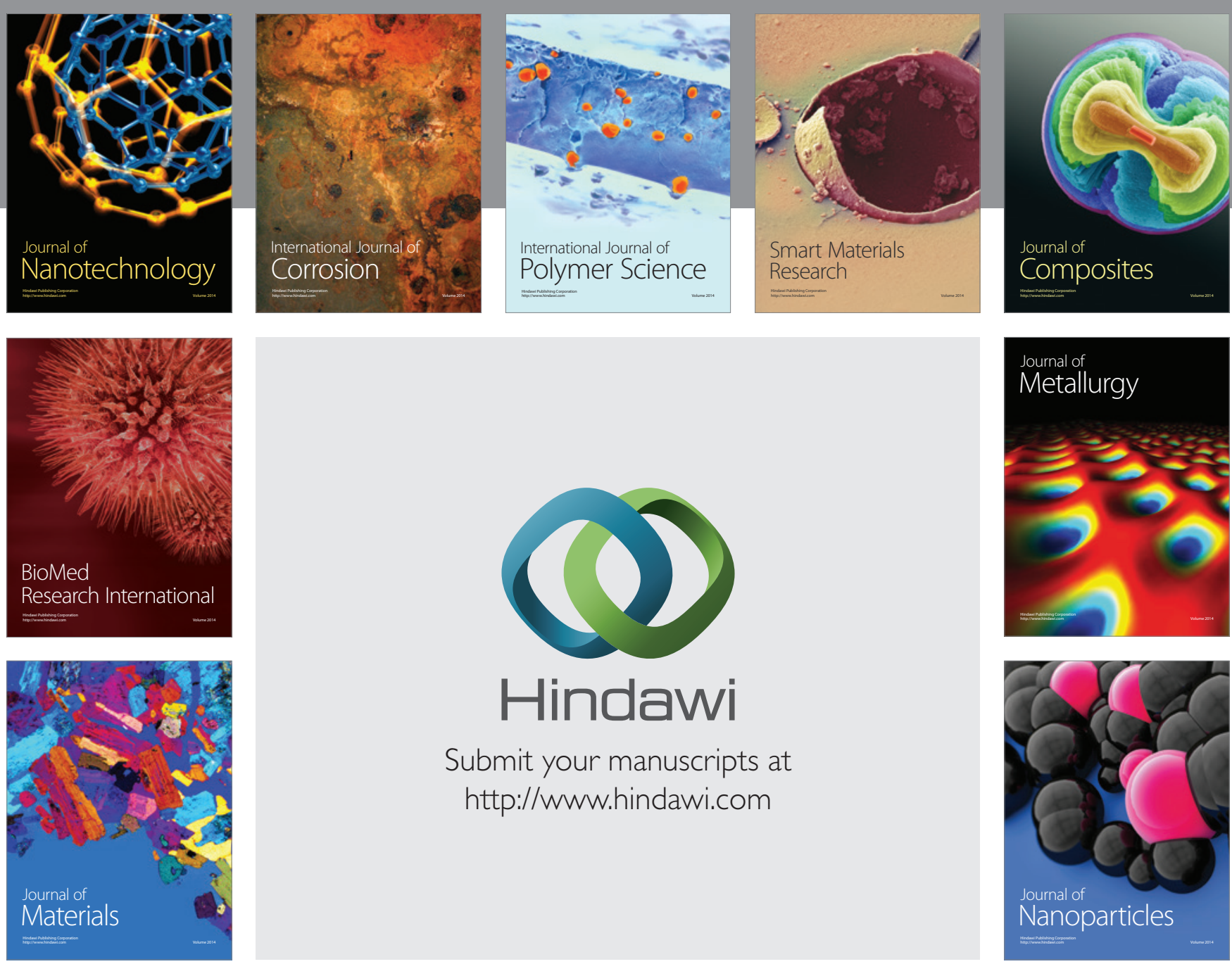

Submit your manuscripts at http://www.hindawi.com
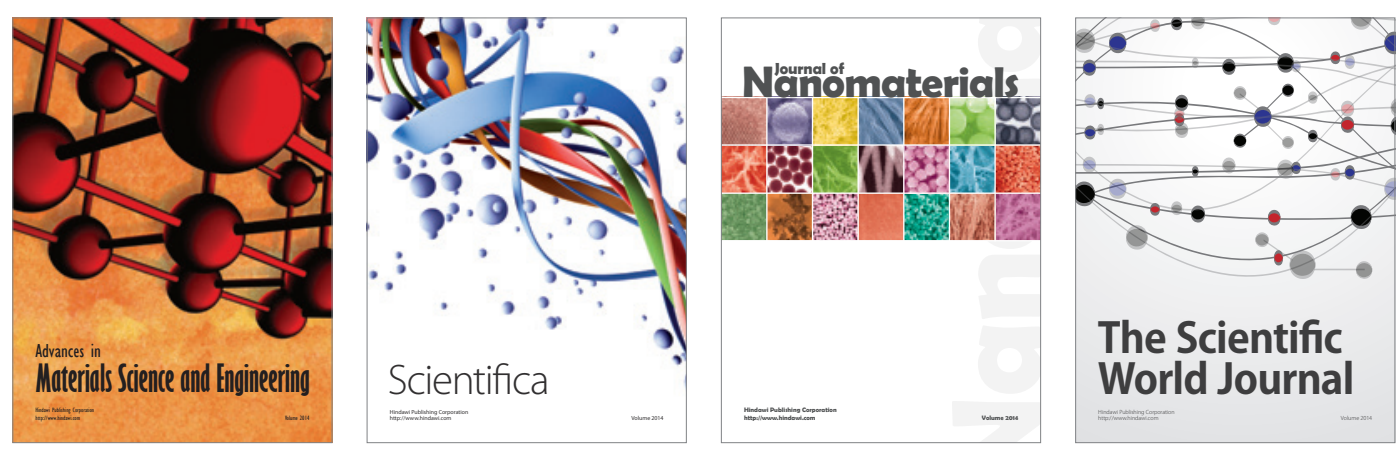

\section{The Scientific World Journal}
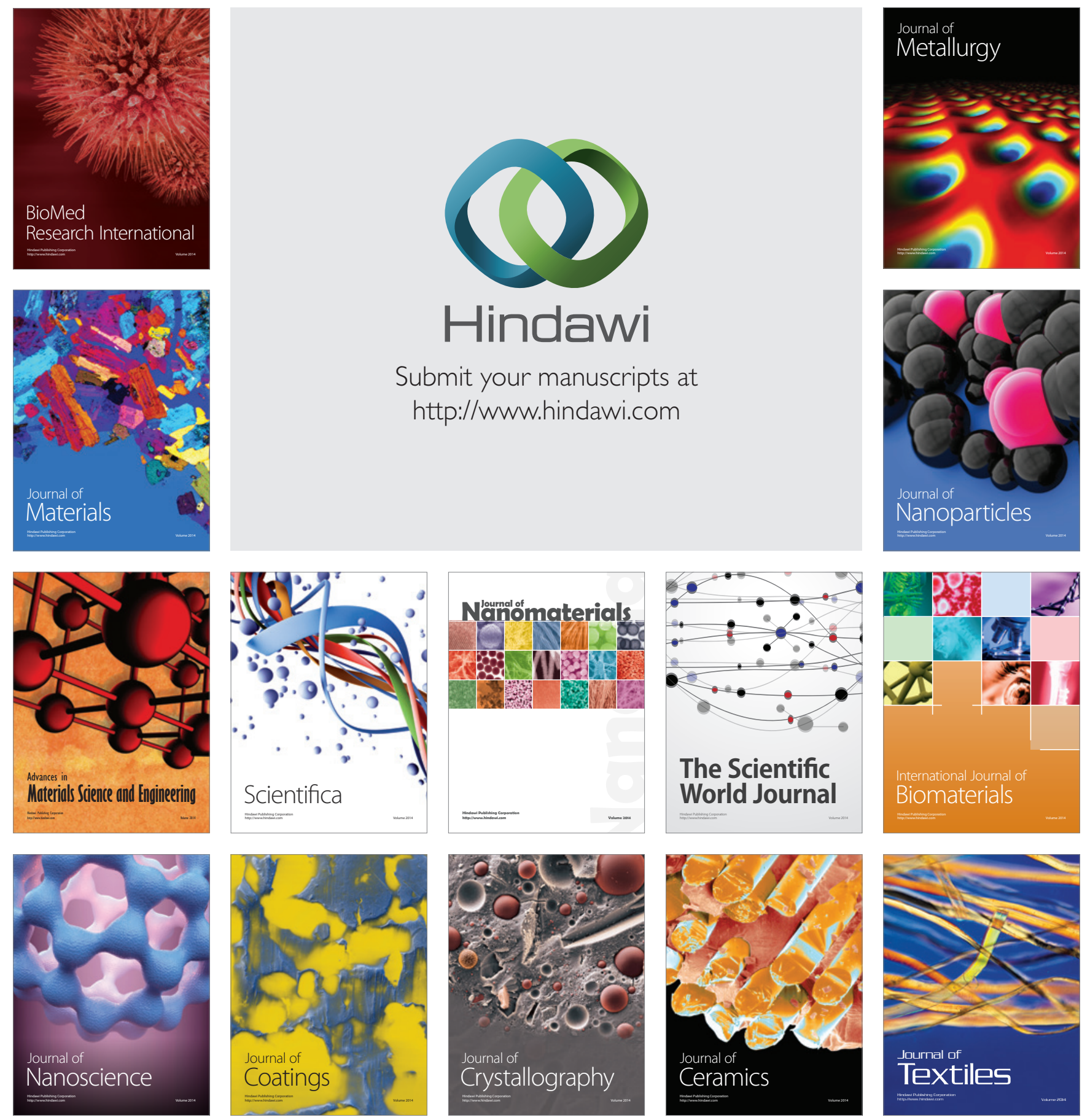